\title{
Case Report: Dynamics of Acquired Fluoroquinolone Resistance under Standardized Short-Course Treatment of Multidrug-Resistant Tuberculosis
}

\author{
Jean Claude Semuto Ngabonziza, ${ }^{1,2,3 \star}$ Armand Van Deun, ${ }^{4}$ Patrick Migambi, ${ }^{5}$ Esdras Belamo Niyigena, ${ }^{1}$ Théogène Dusabe, ${ }^{6}$ \\ Yves Mucyo Habimana, ${ }^{5}$ Bertin Ushizimpumu, ${ }^{1}$ Wim Mulders, ${ }^{2}$ Tom Decroo, ${ }^{7,8}$ Dissou Affolabi, ${ }^{9}$ Philip Supply, ${ }^{10}$ \\ Bouke C. de Jong, ${ }^{2}$ and Leen Rigouts ${ }^{2,3}$ \\ ${ }^{1}$ National Reference Laboratory Division, Department of Biomedical Services, Rwanda Biomedical Centre, Kigali, Rwanda; ${ }^{2}$ Mycobacteriology \\ Unit, Department of Biomedical Sciences, Institute of Tropical Medicine, Antwerp, Belgium; ${ }^{3}$ Department of Biomedical Sciences, University of \\ Antwerp, Antwerp, Belgium; ${ }^{4}$ Independent TB Consultant, Leuven, Belgium; ${ }^{5}$ Tuberculosis and Other Respiratory Diseases Division, Institute of \\ HIVIAIDS Disease Prevention and Control, Rwanda Biomedical Centre, Kigali, Rwanda; ${ }^{6}$ MDR-TB Clinic, Kabutare Hospital, Huye, Rwanda; \\ ${ }^{7}$ Department of Clinical Sciences, Institute of Tropical Medicine, Antwerp, Belgium; ${ }^{8}$ Research Foundation Flanders, Brussels, Belgium; \\ ${ }^{9}$ Laboratoire de Référence des Mycobactéries, Cotonou, Benin; ${ }^{10}$ Univ. Lille, CNRS, Inserm, CHU Lille, Institut Pasteur de Lille,
} U1019-UMR9017-CIIL-Centre d'Infection et d'Immunité de Lille, F-59000 Lille, France

\begin{abstract}
We report a case of acquired fluoroquinolone (FQ) resistance under short-course multidrug-resistant tuberculosis (MDR-TB) treatment. The patient was managed at Kabutare hospital, one of the two specialized MDR-TB clinics in Rwanda. A low dose of moxifloxacin was used in the first three critical months. Acquired resistance was identified at the ninth month of treatment, 3 months after stopping kanamycin in a strain initially susceptible only to FQs, kanamycin, and clofazimine. Fluoroquinolone resistance was detected in the same month by deep sequencing as routinely used second-line line probe assay and phenotypic drug susceptibility testing. High-dose FQ, preferably gatifloxacin, should be used to maximize effectiveness.
\end{abstract}

\section{INTRODUCTION}

Multidrug-resistant tuberculosis (MDR-TB), defined as TB resistant to at least rifampicin and isoniazid, is among the top global challenges in TB control and management. The two standardized WHO-endorsed MDR-TB treatment regimens, that is, a 9-month short treatment regimen (STR) and a 20month regimen, include a later generation fluoroquinolone (FQ) as core drug. The core drug of a regimen is the drug that contributes most to achieving relapse-free cure, whereas companion drugs prevent acquisition of resistance to the core drug, and thus prevent treatment failure. ${ }^{1}$

Fluoroquinolone-resistant MDR-TB in Rwanda is very rare; no cases were identified in the 2015 drug resistance survey ${ }^{2}$ and none during routine testing of more than 400 rifampicin-resistant patients (in place since 2010, National Reference Laboratory Rwanda). ${ }^{3}$ Development of TB drug resistance may be induced by bacterial exposure to low drug levels, as a result of either inappropriately low dosing or suboptimal bioavailability of the drug, yet mainly through unknown resistance profiles to companion drugs, effectively resulting in monotherapy. 4,5

Here, we discuss sequential events preceding the development of FQ resistance in an MDR-TB patient treated with the STR in Rwanda, using targeted deep sequencing (Deeplex-MycTB, GenoScreen, France), with a minimum filtering set at 3\% minority reads, alongside second-line LPA (GenoType MTBDRs/ [Hain Lifescience, Germany]) and phenotypic drug-susceptibility testing (pDST) on subsequent samples to identify the time point at which acquired FQ resistance became detectable.

\section{CASE REPORT}

A 63-year-old, HIV-coinfected woman with no history of TB, diagnosed with rifampicin-resistant TB by Xpert MTB/RIF

*Address correspondence to Jean Claude Semuto Ngabonziza, Kigali, Rwanda. E-mail: jclaude.ngabonziza@rbc.gov.rw
(Cepheid, Sunnyvale, CA), was referred to Kabutare hospital, one of the two MDR-TB-specialized clinics in Rwanda. Clinically, the patient presented with severe symptoms including fever, severe cough, asthenia, thoracic pain, oxygen desaturation (requiring oxygen therapy), weight loss (37 kg; body mass index $=15.4$ ), epigastric pain, and buccal candidiasis. The patient had been taking antiretroviral treatment since 2005 , with 512 CD4 cells/ $\mu \mathrm{L}$ and an undetectable viral load at the start of TB treatment. She was started on the STR 1 day after the diagnosis of rifampicin-resistant TB, as per routine practice in Rwanda. As per national guidelines adapted from a previous survey protocol, ${ }^{6}$ based on the patient's weight, the initiated STR comprised moxifloxacin (200 mg), kanamycin (500 mg), protionamide (500 mg), ethambutol (600 mg), clofazimine (100 mg), isoniazid (300 mg), and pyrazinamide (800 mg).

The second-line LPA, performed on a baseline sample, showed the strain to be susceptible to FQs and second-line injectables. Phenotypic drug-susceptibility testing on the Löwenstein-Jensen (LJ) media confirmed resistance to rifampicin $(40 \mu \mathrm{g} / \mathrm{mL})$, isoniazid $(0.2 \mu \mathrm{g} / \mathrm{mL})$, ethambutol $(2 \mu \mathrm{g} /$ $\mathrm{mL})$, and ethionamide $(40 \mu \mathrm{g} / \mathrm{mL})$, and showed susceptibility to ofloxacin $(2 \mu \mathrm{g} / \mathrm{mL})$ and kanamycin $(30 \mu \mathrm{g} / \mathrm{mL})$. The patient was enrolled in the MDR-TB diagnostic trial "DIAgnostics for MDR-TB in Africa” (DIAMA; Clinicaltrials.gov, NCT03303963). The trial evaluates Deeplex-MycTB, a novel multiplex deep sequencing-based drug resistance diagnostic platform that simultaneously provides sequence information of 18 genes associated with resistance to TB drugs, and new strategies for treatment follow-up. These include fluorescein diacetate (FDA), a vital stain microscopy allowing the distinction between live and dead bacilli. ${ }^{7}$ Routine monitoring included light-emitting diodefluorescence microscopy (FM) and culture on LJ and MGIT media.

At month 4, when the patient's weight reached $>40 \mathrm{~kg}$, the moxifloxacin dose was increased to the standard $400 \mathrm{mg} /$ day in accordance with the National Tuberculosis control Program guideline. Unlike most MDR-TB patients on the STR in Rwanda, among whom culture conversion is usually achieved at the second or third month of treatment, this patient remained 
smear and culture positive at month 4 . Hence, kanamycin was continued for another 2 months (up to the maximum of 6 months). Despite the patient's general improved condition (no fever, no cough, and weight increase to $47 \mathrm{~kg}$ at month 10), she remained smear and culture positive, whereas FDA microscopy smears were negative between months 2 and 6 , yet turned positive again 1 month after stopping kanamycin (Table 1).

As part of routine follow-up testing, samples at months 7 and 8 showed no resistance to second-line drugs. At month 10, FQ resistance was detected by second-line LPA and confirmed by pDST. Repeat second-line LPA and pDST on stored samples and cultures of months 7 and 8 confirmed susceptibility to FQs and second-line injectables, whereas both second-line LPA and PDST revealed FQ resistance on the stored sample and culture of months 9 and 10 (Table 1). At month 11 , the patient was changed to bedaquiline $(400 \mathrm{mg} /$ day for 1 month and $200 \mathrm{mg} \times 3$ per week after 1 month), linezolid (600 mg), and delamanid (100 mg $\times 2 /$ day), while continuing with clofazimine $(200 \mathrm{mg})$, isoniazid $(600 \mathrm{mg})$, and pyrazinamide $(1,200 \mathrm{mg})$. Of note, clinicians were unaware of high-level isoniazid and pyrazinamide resistance as first-line LPA or MGIT-PZA was not done, and sequencing was done à posteriori on stored samples. Despite this change of regimen, the patient's sputum remained smear and culture positive for 5 more months, after which the culture converted to negative from month 16 onward (Table 1).

Consistent with LPA and pDST results, Deeplex-MycTB showed FQ heteroresistance in the sample (57\% gyrA D94G mutant) and culture isolate (63\% D94G mutant) of month 9, yet fully wild-type gyrA profiles in previous samples and isolates, in which the bacterial burden was already increasing. Moreover, Deeplex-MycTB revealed high-level isoniazid resistance associated with combined mutations in the katG gene (S315T) and inhA promoter region (fabG1 T-8C), as well as pyrazinamide (pncA Q10R) resistance in all baseline and follow-up samples and isolates, along with rifampicin (rpoB $\mathrm{S} 450 \mathrm{~L}$ ) and ethionamide resistance (ethA del GTAATTCAAC and inhA promoter mutation, fabG1 T-8C) previously detected by pDST. The strain phylogenetic profile remained unchanged throughout. No mutation was detected in the $r 0678$ or $r$ l targets, associated with resistance to bedaquiline and clofazimine, and linezolid, respectively.

\section{DISCUSSION}

To the best of our knowledge, this is the first study using deep sequencing alongside second-line LPA and pDST to investigate at which timepoint FQ resistance became identifiable in an MDR-TB patient treated with the STR. Despite the ability of Deeplex-MycTB to detect as little as 3\% mutant DNA, heteroresistance to $F Q$ was identified in the same month that pDST and second-line LPA detected FQ resistance. DeeplexMycTB also provided data on mutations in other target genes.

The routinely used second-line LPA and pDST identified FQ resistance at month 10, when the gyrA mutation had become fixed (i.e., at least 95\% mutant DNA). Both second-line LPA and pDST also detected FQ resistance on a stored sample/ isolate, yielding a gyrA mutant subpopulation at month 9, confirming their good detection limit of heteroresistance. ${ }^{8}$ Of note, no culture bias (decreased proportion of mutant bacilli in culture relative to direct testing on sample) was observed, suggesting no substantial fitness cost associated with D94G.
A decade of evidence shows good to excellent performance of the STR, with more than $80 \%$ success rate in different settings including Rwanda. ${ }^{3,6}$ Although the original Bangladesh STR used gatifloxacin as the core drug, nonavailability of this drug urged programs to use moxifloxacin or levofloxacin. However, recent evaluations revealed superior bacteriological effectiveness of the gatifloxacin-based regimen over levofloxacin- or moxifloxacin-based regimens. ${ }^{9}$ Moreover, the rate of $\mathrm{FQ}$ resistance amplification increased from none with gatifloxacin high dose ( $800 \mathrm{mg} /$ day) to $9.9 / 1,000$ and $14-20.3 /$ 1,000 , with very high-dose levofloxacin and standard $400 \mathrm{mg} /$ day or high $(800 \mathrm{mg})$ dose moxifloxacin, respectively, ${ }^{10}$ confirming findings from in vitro and a pharmacodynamic infection model. ${ }^{11,12}$ The highest rate of FQ resistance acquisition was recorded for the Stream randomized clinical trial, even with using $800 \mathrm{mg} /$ day moxifloxacin. ${ }^{13}$ Unfortunately, gatifloxacin is currently unavailable. The manufacturer withdrew the drug from the market following a study that showed that the use of gatifloxacin was associated with dysglycemia among elderly treated for pneumonia. ${ }^{14}$ Our previous experience with gatifloxacin showed that dysglycemia was rare in MDR-TB patients treated with the STR, and, when it occurred, it was manageable. ${ }^{15-17}$

Our patient received the low, weight-adapted dose of $200 \mathrm{mg}$ moxifloxacin per day during the most critical first months. Despite clinical improvement there was no bacteriological clearance for 10 months. Retrospective molecular analysis revealed pyrazinamide, prothionamide and high-level isoniazid resistance in the baseline isolate. In combination with the observed phenotypic ethambutol resistance, protection against acquiring resistance to the core drug mainly depended on the companion drugs kanamycin and clofazimine. ${ }^{1}$ When kanamycin was stopped at 6 months, clofazimine was the only likely active companion drug. This led to a growing bacterial population - as evidenced by the increased FM and FDA positivity from month 7 and 8 onwards respectively. Fluoroquinolone resistance became apparent 3 months after stopping kanamycin, and 5 months after switching to standard $400 \mathrm{mg} /$ day moxifloxacin. Surprisingly, several months before any mutants were detectable the bacterial population increased while undergoing $F Q$ drug pressure. The high FQ-minimal inhibitory concentration values conferred by the D94G mutation $^{12}$ cannot be overcome, even by a high dose.

The use of bedaquiline in patients who have been treated with clofazimine is questioned because of possible effluxpump mediated cross-resistance due to mutations in the $r v 0678$ gene. ${ }^{18}$ Fortunately, and despite the use of clofazimine as the only likely effective companion drug during 6 months, our patient responded well on the bedaquiline-based regimen and was declared cured after 21 months of bedaquiline-based treatment. No mutation was acquired in $r 0678$.

We documented the appearance of FQ resistance in an STR patient initially receiving a low dose of moxifloxacin, shortly after stopping kanamycin. Despite clinical improvement, bacterial clearance was not obtained and FQ resistance developed. FQ resistance was detected in the same month by Deeplex-MycTB as routinely used second-line LPA and pDST. Increasing counts of acid fast bacilli on FM and FDA microscopy were suggestive of treatment failure. Our patient's findings emphasize the importance of using high-dose $F Q$, preferably gatifloxacin, the strongest FQ against TB and the 


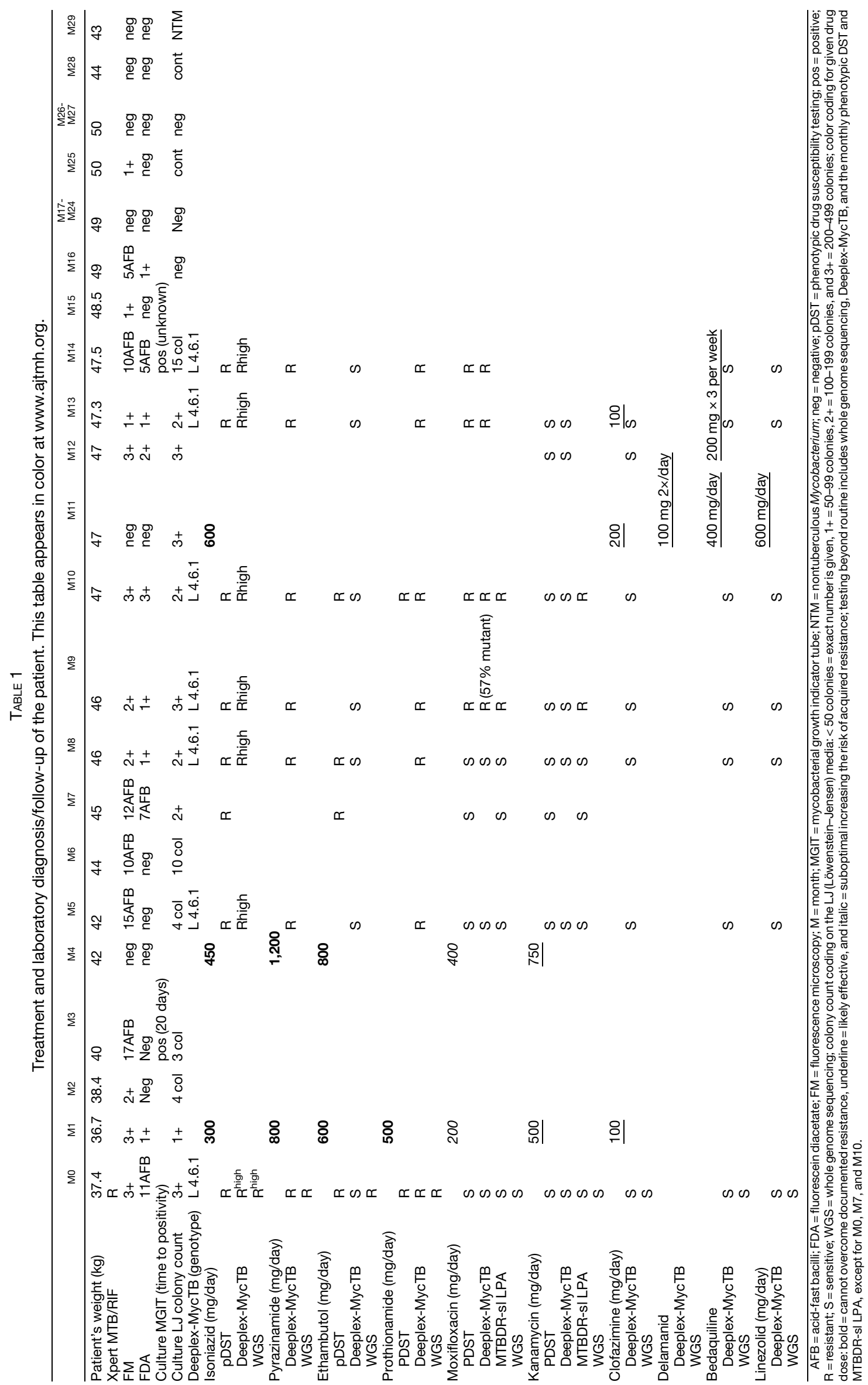


importance of extended molecular and/or pDST at baseline to ensure protection of the core drug effectiveness.

Received March 17, 2020. Accepted for publication May 27, 2020.

Published online July 6, 2020.

Acknowledgments: We thank Maniteze Clemence (Kabutare MDR-TB Clinic) and Musanganire Devotha (Nyanza Health Center) who closely monitored the patient.

Financial support: This publication is based on findings produced in the context of the DIAMA study, which is part of the EDCTP2 program supported by the European Union (Grant number DRIA2014326-DIAMA) and the Belgian Directorate General for Development Cooperation (PhD fellowship to JCSN). P. S. reports personal fees from GenoScreen during the conduct of the study.

Disclaimer: The views and opinions of authors expressed herein do not necessarily state or reflect those of EDCTP.

Authors' addresses: Jean Claude Semuto Ngabonziza, National Reference Laboratory Division, Department of Biomedical Services, Rwanda Biomedical Centre, Kigali, Rwanda, and Mycobacteriology Unit, Department of Biomedical Sciences, Institute of Tropical Medicine, Antwerp, Belgium, E-mail: jclaude.ngabonziza@rbc.gov. rw. Armand Van Deun, Independent TB consultant, Leuven, Belgium, E-mail: avandeun@theunion.org. Patrick Migambi and Yves Mucyo Habimana, Tuberculosis and Other Respiratory Diseases Division, Institute of HIV/AIDS Disease Prevention and Control, Rwanda Biomedical Centre, Kigali, Rwanda, E-mails: patrick.migambi@rbc.gov.rw and yves.mucyo@rbc.gov.rw. Esdras Belamo Niyigena and Bertin Ushizimpumu, National Reference Laboratory Division, Department of Biomedical Services, Rwanda Biomedical Centre, Kigali, Rwanda, E-mails: nbelamo@gmail.com and usbertin1@gmail.com. Théogène Dusabe, MDR-TB Clinic, Kabutare Hospital, Huye, Rwanda, E-mail: dutheos80@gmail.com. Wim Mulders, Bouke C. de Jong, and Leen Rigouts, Mycobacteriology Unit, Department of Biomedical Sciences, Institute of Tropical Medicine, Antwerp, Belgium, E-mails: wmulders@itg.be, bdejong@itg.be, and Irigouts@itg.be. Tom Decroo, Department of Clinical Sciences, Institute of Tropical Medicine, Antwerp, Belgium, and Research Foundation Flanders, Brussels, Belgium, E-mail: tdecroo@itg.be. Dissou Affolabi, Laboratoire de Référence des Mycobactéries, Cotonou, Benin, E-mail: affolabi_dissou@yahoo.fr. Philip Supply, Univ. Lille, CNRS, Inserm, CHU Lille, Institut Pasteur de Lille, U1019-UMR9017CIIL-Centre d'Infection et d'Immunité de Lille, F-59000 Lille, France, E-mail: philip.supply@ibl.cnrs.fr.

This is an open-access article distributed under the terms of the Creative Commons Attribution (CC-BY) License, which permits unrestricted use, distribution, and reproduction in any medium, provided the original author and source are credited.

\section{REFERENCES}

1. Van Deun A, Decroo T, Piubello A, De Jong BC, Lynen L, Rieder $\mathrm{HL}$, 2018. Principles for constructing a tuberculosis treatment regimen: the role and definition of core and companion drugs. Int J Tuberc Lung Dis 22: 239-245.

2. World Health Organization, 2017. Global tuberculosis report 2017: leave no one behind - unite to end TB. Available at: https://www.who.int/campaigns/tb-day/2017/posters/en/.
3. Ngabonziza J-CS et al., 2020. Reduction of diagnostic and treatment delays reduces rifampicin-resistant tuberculosis mortality in Rwanda. Int J Tuberc Lung Dis 24: 329-339.

4. Mitchison DA, 1998. How drug resistance emerges as a result of poor compliance during short course chemotherapy for tuberculosis. Int J Tuberc Lung Dis 2: 10-15.

5. Zhang Y, Yew WW, 2015. Mechanisms of drug resistance in Mycobacterium tuberculosis: update 2015. Int J Tuberc Lung Dis 19: 1276-1289.

6. Schwœbel V et al., 2020. Outcomes of a nine-month regimen for rifampicin-resistant tuberculosis up to 24 months after treatment completion in nine African countries. EClinicalMedicine 20: 100268.

7. Van Deun A, Maug AKJ, Hossain A, Gumusboga M, De Jong BC, 2012. Fluorescein diacetate vital staining allows earlier diagnosis of rifampicin-resistant tuberculosis. Int $J$ Tuberc Lung Dis 16: 1174-1179.

8. Rigouts L, Miotto P, Schats M, Lempens P, Cabibbe AM, Galbiati S, Lampasona V, de Rijk P, Cirillo DM, de Jong BC, 2019. Fluoroquinolone heteroresistance in Mycobacterium tuberculosis: detection by genotypic and phenotypic assays in experimentally mixed populations. Sci Rep 9: 11760.

9. Van Deun A, Decroo T, Kuaban C, Noeske J, Piubello A, Aung KJM, Rieder HL, 2019. Gatifloxacin is superior to levofloxacin and moxifloxacin in shorter treatment regimens for multidrugresistant TB. Int J Tuberc Lung Dis 23: 965-971.

10. Trébucq A, Decroo T, Deun AV, Piubello A, Chiang C, Koura KG, Schwoebel V, 2020. Short-course regimen for multidrugresistant tuberculosis: a decade of evidence. J Clin Med 9: 55.

11. Gumbo T, Louie A, Deziel MR, Parsons LM, Salfinger M, Drusano GL, 2004. Selection of a moxifloxacin dose that suppresses drug resistance in Mycobacterium tuberculosis, by use of an in vitro pharmacodynamic infection model and mathematical modeling. J Infect Dis 190: 1642-1651.

12. Rigouts $L$ et al., 2016. Specific gyrA gene mutations predict poor treatment outcome in MDR-TB. J Antimicrob Chemother 71: 314-323.

13. Nunn AJ et al., 2019. A trial of a shorter regimen for rifampinresistant tuberculosis. N Engl J Med 380: 1201-1213.

14. Chiang CY, Van Deun A, Rieder HL, 2016. Gatifloxacin for short, effective treatment of multidrug-resistant tuberculosis. Int $J$ Tuberc Lung Dis 20: 1143-1437.

15. Aung KJM, Van Deun A, Declercq E, Sarker MR, Das PK, Hossain MA, Rieder HL, 2014. Successful "9-month Bangladesh regimen" for multidrugresistant tuberculosis among over 500 consecutive patients. Int J Tuberc Lung Dis 18: 1180-1187.

16. Kuaban C, Noeske J, Rieder HL, Aït-Khaled N, Abena Foe JL, Trébucq A, 2015. High effectiveness of a 12-month regimen for MDR-TB patients in Cameroon. Int J Tuberc Lung Dis 19: 517-524.

17. Piubello A, Harouna SH, Souleymane MB, Boukary I, Morou S, Daouda M, Hanki Y, Van Deun A, 2014. High cure rate with standardised short-course multidrugresistant tuberculosis treatment in Niger: no relapses. Int J Tuberc Lung Dis 18: 1188-1194.

18. Hartkoorn RC, Uplekar S, Cole ST, 2014. Cross-resistance between clofazimine and bedaquiline through upregulation of mmpl5 in mycobacterium tuberculosis. Antimicrob Agents Chemother 58: 2979-2981. 